\title{
Q-Switched Laser Therapy for Recalcitrant and Recurrent Pigmentation After Chemical Depigmentation for Extensive Vitiligo
}

\author{
Alison B. Durham, MD, and JefFrey S. Orringer, MD*
}

The authors have indicated no significant interest with commercial supporters.

$\mathrm{V}$ itiligo is an acquired, idiopathic disease characterized by depigmented macules and patches, with total absence of functional melanocytes within the areas of affected skin. This is a condition that occurs worldwide, with an estimated prevalence of $1 \%$ but with reported rates as high as $4 \%$ in some countries. Although spontaneous remission may occur, given that the condition is associated with significant emotional distress for patients, treatment of the condition is indicated. ${ }^{1}$

Treatment of vitiligo is generally divided into two categories - therapies aimed at repigmentation of affected skin and therapies aimed at depigmentation of unaffected skin for blending of overall skin tones. Depigmentation is often preferred in extensive cases in which vitiligo affects more than $50 \%$ of the total body surface area. Depigmentation may be achieved through the use of topical 20\% monobenzyl ether of hydroquinone $(\mathrm{MBEH}), \mathrm{MBEH}$ in combination with tretinoin, 4-methoxyphenol, $88 \%$ phenol solution, cryotherapy, or laser therapy. ${ }^{1}$ The $694-n m$ qualityswitched (QS) ruby and the 755-nm QS alexandrite lasers have been shown to be effective for depigmentation of recurrent or resistant relative hyperpigmentation in patients with vitiligo. ${ }^{1,2}$ In this case, the 532-nm QS frequency-doubled neodymium- doped yttrium aluminum garnet (Nd:YAG) laser was used successfully to treat areas of recalcitrant pigmentation and recurrent pigmentation after fullbody chemical depigmentation therapy for vitiligo.

\section{Case Report}

A 23-year-old woman of Middle Eastern descent with baseline Fitzpatrick type III skin presented to our clinic for treatment of patchy areas of relative hyperpigmentation involving her face, arms, trunk, and legs that had been present for longer than 1 year. She had a history of extensive vitiligo beginning at the age of 8 that failed to repigment after multiple treatment attempts. She underwent total body chemical depigmentation with $20 \%$ $\mathrm{MBEH}$ at the age of 12 , with generally excellent results aside from areas of recalcitrant pigmentation on the extremities. The patient continued to use topical MBEH therapy intermittently for the next 4 years to treat the persistent pigmentation and areas of sun-induced repigmentation. Eventually, she began to note areas of repigmentation that were resistant to further attempts at chemical depigmentation. She presented to our clinic for a discussion of other therapeutic options. Examination at that time revealed Fitzpatrick skin type I with blotchy, mac-

*Both authors are affiliated with the Department of Dermatology, University of Michigan Medical School, Ann Arbor, Michigan 


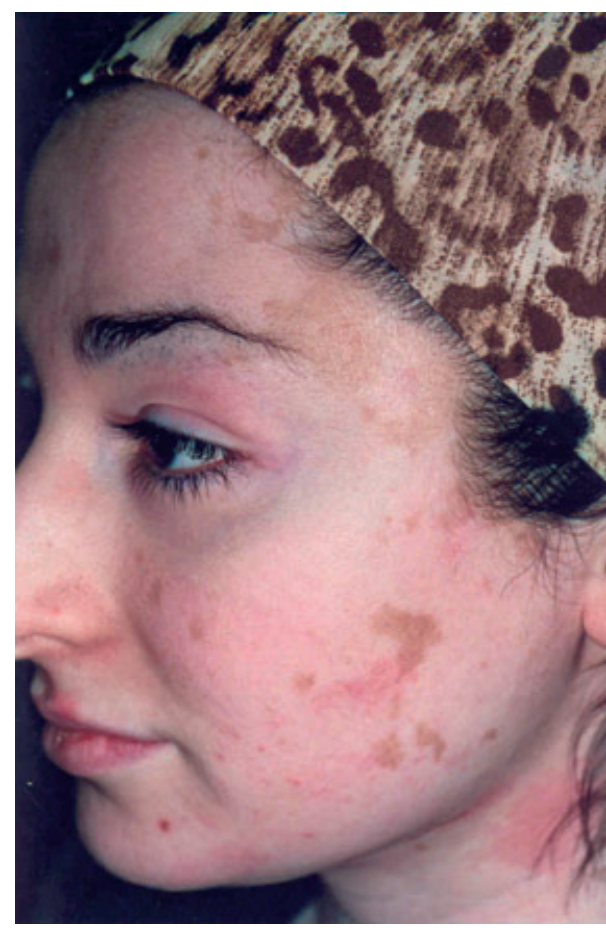

Figure 1. Blotchy macular hyperpigmentation of the patient's face secondary to recurrent pigmentation after treatment with topical $20 \%$ monobenzyl ether of hydroquinone.

ular, light brown relative hyperpigmentation scattered over the forehead, temples, cheeks, legs, and arms (Figure 1).

After confirmation of efficacy on a test spot, the patient was treated serially with nonoverlapping pulses of a 532-nm QS Nd:YAG laser with a 3- to 4-mm spot size and a fluence of 1.1 to $1.8 \mathrm{~J} / \mathrm{cm}^{2}$ followed by application of petrolatum several times daily for approximately 7 days. Twice-daily applications of $4 \%$ hydroquinone cream were begun approximately 1 week after treatment, and she also began strict and continuous daily sunscreen use perioperatively except during the first week after each treatment because of concerns about potential skin irritation. This overall treatment protocol produced excellent results (Figure 2). Aside from the expected purpura secondary to the laser therapy, she experienced no side effects from the procedure during any of her treatment sessions. Each treated site generally responded completely after one treatment session,

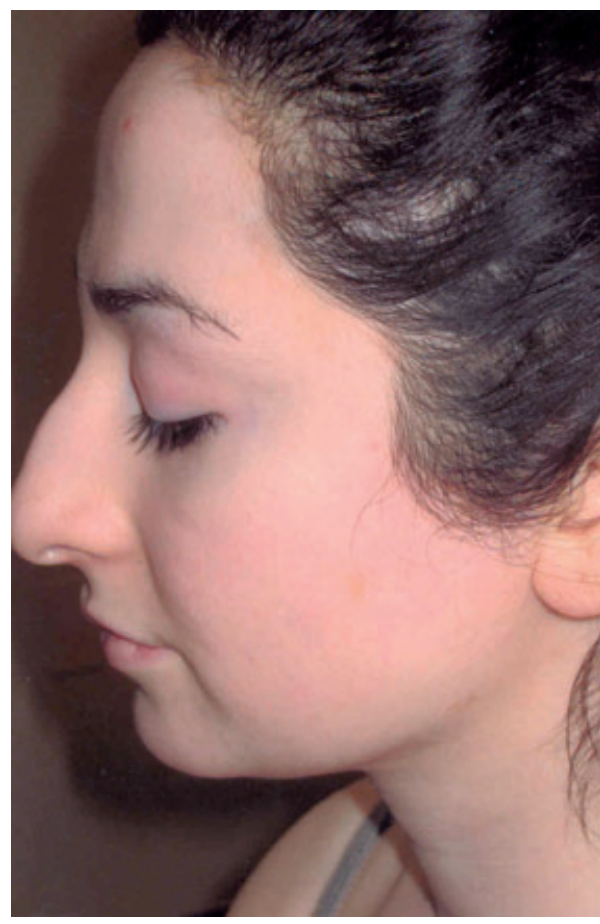

Figure 2. Clearance of hyperpigmentation several weeks after a single quality-switched frequency-doubled neodymium-doped yttrium aluminum garnet laser treatment session.

but any residual relative hyperpigmentation was treated every 6 to 8 weeks until it resolved.

Over the past 7 years, the patient has been treated intermittently on an as-needed basis, typically requiring treatment of new breakthrough repigmentation no more than once per year. Treated sites have included the face, trunk, and all extremities. In most cases, treated sites have not repigmented over a period of several years.

\section{Discussion}

Treatment of extensive vitiligo by removing residual pigmentation can be an effective way to improve cosmesis in patients who fail to respond to therapies aimed at repigmentation of affected skin or in patients with more than $50 \%$ total body surface area involvement. One of the most widely used means of achieving depigmentation is with twice-daily application of $20 \% \mathrm{MBEH}^{1}{ }^{1}$ This treatment must gener- 
ally be continued for many months and has a relatively high failure rate. One retrospective review of 18 patients showed that complete depigmentation was achieved in only eight. The degree of depigmentation achieved correlated with duration of use, sometimes requiring use for 10 months or more to achieve complete depigmentation. ${ }^{3}$ The preparation may be difficult to tolerate because it causes ocular and cutaneous chemical irritation. ${ }^{1}$ Additionally, areas of skin may repigment at a later date, leaving the patient cosmetically disfigured. ${ }^{3}$

Options such as cryotherapy and laser therapy that target melanocytes for destruction have been shown to be effective for removal of residual pigmentation not responsive to additional chemical depigmentation or for patients unable to tolerate the side effects of chemical depigmentation. ${ }^{1,2}$ Additionally, although the Koebner phenomenon may be more common in progressive vitiligo than stable vitiligo, ${ }^{4}$ treatments such as laser therapy may initiate a Koebner response in the affected areas, making them even more amenable to laser treatment than other pigmented lesions. $^{2}$

The most widely reported lasers used for this purpose are the QS ruby $(694 \mathrm{~nm})$ and alexandrite (755 nm) lasers. ${ }^{1,2}$ Although the 532-nm QS Nd-

YAG laser has been shown to be effective for treatment of various pigmented lesions, ${ }^{5}$ to our knowledge, there are no reports of it being used to treat residual or recurrent relative hyperpigmentation in vitiligo.
We present this case to illustrate the efficacy of the 532-nm QS Nd:YAG laser in the treatment of pigmentary disturbances in vitiligo, providing another therapeutic option for this distressing disorder. Our patient tolerated laser treatment well, with no side effects. The patient has intermittently required additional treatment of newly pigmented areas but has maintained long-lasting results with excellent cosmesis after this therapy.

\section{References}

1. Al Ghamdi KM, Kumar A. Depigmentation therapies for normal skin in vitiligo universalis. J Eur Acad Dermatol Venereol 2011;25:749-57.

2. Thissen M, Westerhof W. Laser treatment for further depigmentation in vitiligo. Int J Dermatol 1997;36:386-8.

3. Mosher DB, Parrish JA, Fitzpatrick TB. Monobenzylether of hydroquinone: a retrospective study of treatment of 18 vitiligo patients and a review of the literature. Br J Dermatol 1977;97: 669-79.

4. Njoo MD, Das PK, Bos JD, Westerhof W. Association of the Köbner phenomenon with disease activity and therapeutic responsiveness in vitiligo vulgaris. Arch Dermatol 1999;135: 407-13.

5. Bowes LE, Nouri K, Berman B, Jimenez G, et al. Treatment of pigmented hypertrophic scars with the $585 \mathrm{~nm}$ pulsed dye laser and the $532 \mathrm{~nm}$ frequency-doubled Nd:YAG laser in the Qswitched and variable pulse modes: a comparative study. Dermatol Surg 2002;28:714-9.

Address correspondence and reprint requests to: Jeffrey S. Orringer, MD, University of Michigan Medical School, 1500 E. Medical Center Drive, Department of Dermatology, 1910 Taubman Center, Ann Arbor, MI 48109, or e-mail: jorringe@umich.edu 